\title{
Role of Traditional \& Religious Leaders in Stigma Reduction
}

\author{
Janice L Cooper* and CC Benedict Dossen** \\ The Carter Center, Liberia Mental Health Team, West Africa
}

*Corresponding author: Janice L Cooper PhD, The Carter Center Liberia Mental Health Team, Monrovia, Liberia, West Africa.

CC Benedict Dossen, MS Program Lead, The Carter Center, Monrovia, Liberia, West Africa.

Received Date: July 06, 2019

Published Date: July 12, 2019

\section{Commentary}

I bring to your attention an issue in mental health that gets scarce attention in the peer- reviewed literature: the use of traditional and religious healing in mental health. Funding for mental health services and consequently mental health services research in low-and income countries remains low compared to other health funding. Funding to examine the role of traditional and religious healing in mental health is even smaller. A systematic review of research involving religious and traditional healing in mental health included 8 studies from Sub-Saharan Africa spanning from 1999-2013 [1]. The number of participants in these studies ranged from 59-129. Recently, the National Institute of Mental Health of the United States government funded a study under the direction of Dr. Oye Gureje that examines training and supports for religious and traditional providers who address mental illness and epilepsy [2]. The PAM-D study, a NIMH initiative, examined the integration of mental health treatment options from a combined bio-medical, traditional and faith-healing perspective [3].

A World Bank-funded project in Liberia through the Carter Center Mental Health Program with which I have been engaged was a post-Ebola initiative that sought to address the immediate mental health and psychosocial impact of the epidemic in Liberia from 2015-2018. The program "Supporting Psychosocial Health and Resilience" included training of health care providers and an anti-stigma program. The role that traditional and religious leaders played in the Ebola outbreak, has been recognized both in terms of reducing and even stopping the spread, as well as, sometimes, particularly initially, as potential drivers of the spread of the epidemic [4]. One component The Carter Center's work with the Ministry of Health was to enlist religious and traditional leaders in primary mental health identification, referral and anti- stigma work. The program sought to support 200 religious and traditional leaders in two highly EVD-affected counties with: i) education on mental health and mental illness in Liberia; ii) information on how to identify acute mental illness and where to refer for communitybased mental health services; iii) which mental health specialists could be contacted in mental health emergencies and referral points; and, iv) training on how to reduce stigma. We conducted a study with a subset of those leaders. The thirty-five respondents who participated in the study were religious and traditional leaders. Each respondent provided informed consent.

Participants were trained in skills to identify acute mental illness and referrals to community mental health services, how to differentiate between different types of mental illness, how to collaborate with mental health provirus, how to mobilize communities to support persons with mental illness and how to train and work to reduce stigma against persons with mental illness and epilepsy. Trainers were mental health clinicians, social workers and managers of county mental health and social work services. Trainees received training on common mental health conditions, anti-stigma, human rights of persons with mental illness, and on the referral pathway. This training was augmented with monthly group meetings, and planning meetings for World Mental Health Awareness Day. We conducted a study on the intervention's effectiveness. Key informants, who were a selected from the trained faith/religious and traditional leaders, perceived mobilization (30\%), counseling (30\%) and stigma reduction (20\%) as the most effective factors in addressing mental health during the Ebola outbreak and the country's recovery. The vast majority of referrals to county health authorities were from religious leaders (78\%) compared to traditional leaders (21\%). The University of Liberia UL-PIRE IRB approved this study. 
Studies like this and others that report on programs that seek the bridge the gap between religious and traditional healing and public health and bio-medically-based mental health treatment and supports may significantly increase access to care for persons with mental health conditions, diminish stigma and reduce suffering and the burden of care.

\section{Recognition}

Chris Slotta MPH, the County Health Teams of Montserrado and Margibi Counties and the Ministry of Health of Liberia.

\section{Acknowledgment}

None.

\section{Conflict of interest}

Authors declare no conflict of interest.

\section{References}

1. Nortie G, Oladeji B, Gureje O, Seedat S (2016) Effectiveness of Traditional Healers in Treating Mental Health Disorders: A Systematic Review. Lancet Psychiatry 3(2): 154-170.

2. Partnership for Mental Health Development in Sub-Saharan Africa.

3. Op.cit.

4. Manguvo A, Mafuvadze B (2015) The Impact of Traditional and Religious Practices on the Spread of Ebola in West Africa: Time for a Strategic Shift. Pan Afr Med J 22(Supplemental 1): 9. 